\title{
A Case of Ramsay Hunt Syndrome with Cranial Polyneuropathy
}

\author{
Chang-Hee Kim¹, Seong il Kang ${ }^{2}$ and Young Ho Kim² \\ ${ }^{1}$ Department of Otorhinolaryngology-Head and Neck Surgery, Konkuk University Medical Center, \\ Konkuk University School of Medicine, Seoul, \\ ${ }^{2}$ Department of Otorhinolaryngology-Head and Neck Surgery, Seoul Metropolitan Government, Seoul National University, \\ Boramae Medical Center, Seoul, Korea
}

Received December 27, 2011

Revised March 15, 2012

Accepted April 6, 2012

Address for correspondence

Young Ho Kim, MD

Department of Otorhinolaryngology-

Head and Neck Surgery,

Seoul Metropolitan Government,

Seoul National University,

Boramae Medical Center,

20 Boramae-ro 5-gil, Dongjak-gu,

Seoul 156-707, Korea

Tel $+82-2-870-2442$

Fax $+82-2-870-3863$

E-mail yhkiment@gmail.com
Ramsay Hunt syndrome is an infectious disease caused by the varicella zoster virus. It is characterized by the symptoms of facial paralysis, otalgia, and erythematous vesicular rash on the auricle and/or oral cavity. We present a case of a patient with clinical evidence of Ramsay Hunt syndrome involving the cranial nerves VI, VII, and VIII. The patient, a 66-year-old woman, showed left facial palsy associated with vesicular eruptions on her left auricle, impaired gaze to left side, and left hearing loss. Four months after the treatment with systemic steroid and antiviral agents, her all symptoms and signs including facial paralysis and diplopia disappeared.

Korean J Audiol 2012;16:80-82

KEY WORDS: Ramsay Hunt syndrome · Cranial polyneuropathy · Steroid.

\section{Introduction}

Varicella zoster virus (VZV) infection is an important cause of cranial neuropathy. A wide spectrum of diseases including shingles and chickenpox can be caused by VZV. Ramsay Hunt syndrome (RHS), first described by J. Ramsay Hunt in 1907, refers to the association of unilateral peripheral facial nerve palsy (FNP) and reactivation of VZV along the sensory nerves innervating the ear (herpes zoster oticus). ${ }^{1)}$ Occasionally simultaneous involvement of multiple cranial nerves by VZV can induce symptoms of neurological deficit including impaired eye movement, trigeminal neuralgia, cochleovestibular symptoms, swallowing difficulty, hoarseness, or tongue deviation. ${ }^{2)}$ There have been several case reports of RHS with cranial polyneuropathy $(\mathrm{CP})$, but it still remains to be clarified whether RHS associated with $\mathrm{CP}$ has a poorer prognosis compared with RHS alone. Previously, we reported a series of cases with RHS presenting as $\mathrm{CP}$, in which the most commonly involved cranial nerves (in descending order) were VII, VIII, IX, X, V and III/XII. ${ }^{2)}$ We found that RHS associated with CP has a good prognosis for FNP and other cranial nerve palsies when treated with a combination therapy of systemic antiviral agents and steroids, but not for recovery of hearing loss. ${ }^{2)}$ Here, we report another patient with RHS presenting as CP involving cranial nerves VI, VII, and VIII.

\section{Case Report}

A 66-year-old female was referred to our clinic with left facial palsy, diplopia, and vertigo six days after the onset of left ear pain and headache. The patient underwent a severe emotional stress due to her sister's death at the time of onset of left ear pain. The clinical manifestations developed gradually over a few days. Physical examinations revealed vesicular eruptions with crusts on left auricle, left facial palsy, left abducens nerve palsy, and mild right beating spontaneous nystagmus. The degree of FNP was House-Brackmann (HB) grade V (Fig. 1). Diplopia was present in the primary position and was more prominent on attempted abduction of the left eye (Fig. 2A). Audiogram showed sensorineural hearing loss on her left ear (Fig. 3). Vestibular function test was performed on the fourth hospital day when the patient no longer complained of dizziness, and videonystagmography showed no evidence of spontaneous nystagmus. The size and response to light were 

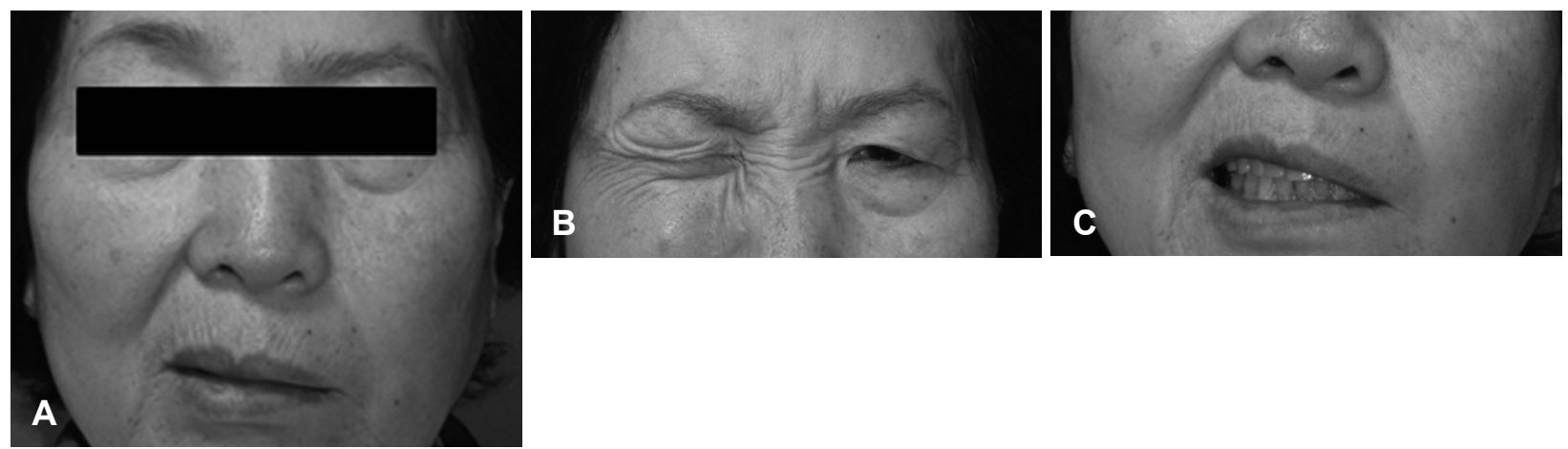

Fig. 1. Obvious asymmetry was observed during resting (A), eye closure (B), and smiling (C).
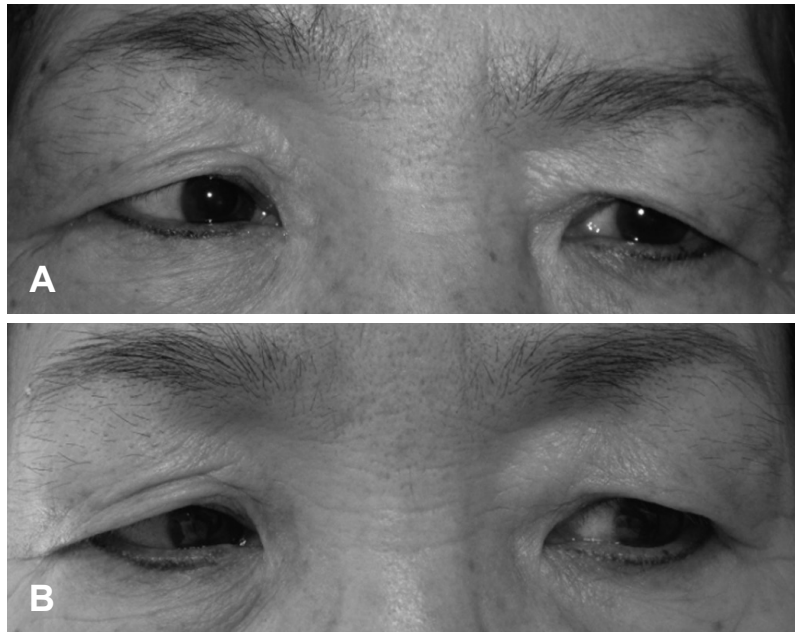

Fig. 2. A: Incomplete abduction of the left eye during attempted left gaze. B: Complete improvement of the left cranial nerve VI palsy is seen 4 months after the combination therapy of steroid and antiviral agents.

normal on both pupils. Except for cranial nerves VI, VII, and VIII, examination for other cranial nerves revealed intact function. Magnetic resonance imaging showed no sign of tumor, infection or other demyelinating diseases of the brain. Serology results for VZV IgG antibodies were positive, but negative for VZV IgM antibodies. The patient had no associated immunocompromised condition including immunosuppressant drug use or immunodeficiency syndrome. A diagnosis of RHS with CP was made based on the patient's characteristic presentation, and from a clinical point of view cranial nerves VI, VII, and VIII were found to be involved. Systemic administration of steroid and antiviral agents was started. Sixty mg of prednisolone was initially given to the patient and tapered orally for 14 days, and $3000 \mathrm{mg}$ of acyclovir was administered intravenously for 7 days. No immediate or delayed side effects were observed during and after the treatment. Among symptoms, dizziness improved most rapidly within several days. During 3 weeks from the start of treatment a gradual improvement of the facial weakness and diplopia was observed. Four months after the treatment, FNP recovered to HB grade I and the abducens nerve palsy completely improved without diplopia (Fig. 2B).

\section{Discussion}

RHS has been reported to have the incidence of 5 per 100000 , and known to be approximately $12 \%$ of all FNP cases. ${ }^{3)}$ RHS is characterized by a poor prognosis and high rate of sequelae even after the treatment. Originally, the involvement of cranial nerve VII with otalgia, cutaneous and mucosal lesion, and facial weakness was described for RHS by Hunt ${ }^{4}$ in 1907, and subsequently, he described hearing loss, tinnitus, vertigo, and nystagmus involving cranial nerve VIII. Thus, RHS is an expression of involvement of two cranial nerves (VII and VIII) which are adjacent to each other. Hunt postulated that the syndrome is caused by a reactivation of VZV in the ganglion of cranial nerve VII, ${ }^{4)}$ which was later confirmed by demonstration of presence of VZV DNA in the geniculate ganglion. ${ }^{5}$

The nuclei for cranial nerves III through XII are contained in the brainstem with the close anatomical localization, which may increase the possibility of comorbidity of cranial nerves in RHS with CP. It was previously reported that the most commonly involved cranial nerves (in descending order) were VII, VIII, IX, V, X, and VI. ${ }^{6}$ Our recent study of 11 cases demonstrated the involvement of cranial nerves VII, VIII, IX, X, V and III/XII in this order, and revealed a good prognosis for FNP and other cranial nerve palsies with a combination therapy of steroids and antiviral agents, but not for recovery of hearing loss. ${ }^{2)}$ Several mechanisms of multiple cranial nerve involvement due to a reactivation of VZV have been proposed. Hunt $^{7)}$ explained the mechanism of multiple cranial nerve palsy as an involvement of adjacent ganglia by contiguous anatomical contact from the original source of inflammation in a single ganglion. Another theory was that the spread of the virus 
Fig. 3. Pure tone audiometry showed sensorineural hearing loss on the patient's left ear.

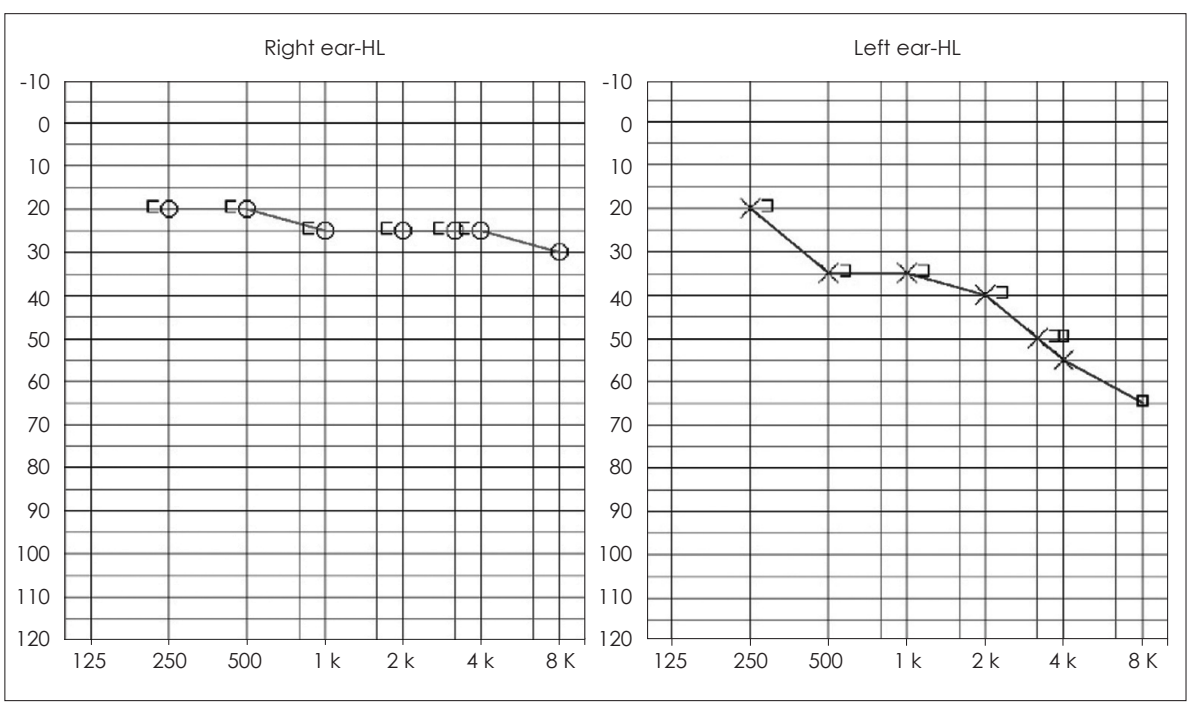

through a common blood supply to cranial nerves might be the mechanism of multiple cranial nerve involvement. ${ }^{1)} \mathrm{Be}-$ sides cranial nerves, other peripheral or central involvements associated with RHS have been reported including Horner's syndrome, herpes zoster uveitis, meningitis, and the syndrome of inappropriate secretion of antidiuretic hormone. ${ }^{6,8,9)}$

A ganglion of cranial nerve VI is in close anatomical proximity to ganglion of cranial nerve VII or VIII. The patient of this case showed the vestibular symptom possibly induced by the involvement of vestibular nerve in cranial nerve VIII. It has been reported that the incidence of vestibular nerve involvement is three- to four-fold higher than that of cochlear nerve involvement when cranial nerve VIII is associated with RHS. ${ }^{10)}$ Vertigo in this case showed the earliest improvement among symptoms, which may be thought to be enhanced by central mechanism such as vestibular compensation. Cranial nerve VI palsy is the most common cranial nerve palsy involved in the dysfunction of ocular mobility, and affected patients suffer from horizontal diplopia. The symptom becomes worse when looking to the affected side, and often the patients turn their face to the affected side.

Correct diagnosis and early treatment of RHS with CP is essential since severe sequelae can be left. The associated complications of cranial polyneuropathy might lead to life-threatening conditions such as a serious acute respiratory distress. Moreover, in case of widespread involvement of unilateral cranial nerves, the possibility of malignant infiltration or sarcoidosis should be considered. Although the beneficial evidence of antiviral therapy in RHS still remains controversial, more aggressive managements including systemic administration of antiviral agents might be required in cases of RHS with CP. The combination therapy with high dose prednisolone for
2 weeks and acyclovir for 1 week showed satisfactory treatment results in our previous study. ${ }^{2)}$ In this case, all symptoms and signs of FNP, diplopia, and vertigo improved after treatment. Early initiation of combination therapy with high dose steroids and antiviral agents for sufficient period immediately after onset of symptoms might result in the good treatment outcome.

\section{REFERENCES}

1) Sweeney CJ, Gilden DH. Ramsay Hunt syndrome. J Neurol Neurosurg Psychiatry 2001;71:149-54.

2) Kim YH, Chang MY, Jung HH, Park YS, Lee SH, Lee JH, et al. Prognosis of Ramsay Hunt syndrome presenting as cranial polyneuropathy. Laryngoscope 2010;120:2270-6.

3) Peitersen E. Bell's palsy: the spontaneous course of 2,500 peripheral facial nerve palsies of different etiologies. Acta Otolaryngol Suppl 2002:4-30.

4) Hunt JR. On herpetic inflammation of the geniculate ganglion: a new syndrome and its complications. J Nerv Ment Dis 1907;34:7396.

5) Van de Steene V, Kuhweide R, Vlaminck S, Casselman J. Varicella zoster virus: beyond facial paralysis. Acta Otorhinolaryngol Belg 2004;58:61-6

6) Aviel A, Marshak G. Ramsay Hunt syndrome: a cranial polyneuropathy. Am J Otolaryngol 1982;3:61-6.

7) Hunt JR. The symptom-complex of the acute posterior poliomyelitis of the geniculate, auditory, glossopharyngeal and pneumogastric ganglia. Arch Intern Med 1910;5:631-75.

8) Kageyama Y, Nakamura M, Sato A, Sato M, Nakayama S, Komatsuzaki O, et al. Syndrome of inappropriate secretion of antidiuretic hormone (SIADH) associated with Ramsay Hunt syndrome: report of a case and review of the literature. Jpn J Med 1989;28:219-22.

9) Bhattacharyya PC, Kakati S. Ramsay Hunt syndrome with aseptic meningitis. J Assoc Physicians India 1993;41:113-4.

10) Turner JE, Geunes PM, Schuman NJ. Cranial polyneuropathy-Ramsay Hunt's syndrome: case report and discussion. Oral Surg Oral Med Oral Pathol Oral Radiol Endod 1997;83:354-7. 